\title{
Current-driven Skyrmion dynamics along curved tracks
}

\author{
Ricardo Francisco Luis ${ }^{1}$, Víctor Raposo ${ }^{1}$, Óscar Alejos ${ }^{2}$, Eduardo Martínez ${ }^{1}$ \\ ${ }^{1}$ Departamento de Física Aplicada, University of Salamanca, Salamanca E-37008, Spain. \\ ${ }^{2}$ Departamento de Electricidad y Electrónica, University of Valladolid, Salamanca E-47011, Spain.
}

\begin{abstract}
The current-driven skyrmion motion along two exchange-coupled ferromagnetic layers with perpendicular magnetic anisotropy is studied by means of micromagnetic simulations and compared to the conventional case of a single ferromagnetic layer. Our results indicate that the two coupled skyrmions can be synchronously driven along the each ferromagnetic layer in the presence of a strong interlayer exchange coupling, and that the velocity is significantly enhanced due to the antiferromagnetic exchange coupling as compared with the single ferromagnetic layer case. The interfacial Dzyaloshinskii-Moriya interaction gives the required chirality to the magnetization textures, while the interlayer exchange coupling favors the synchronous movement of the coupled skyrmions by a dragging mechanism, without depicting the unwanted skyrmion Hall effect. This observation is particularly relevant to drive skyrmions along curved strips, which are also evaluated here. These results indicate that the antiferromagnetic coupling between the ferromagnetic layers mitigates the skyrmion Hall effect, which suggests these systems to achieve efficient and highly-packed displacement of trains of skyrmions for spintronics devices. A study taking into account defects and thermal fluctuations allows to analyze the validity range of these claims.
\end{abstract}

Index Terms-Skyrmions, Spin Hall effect, Dzyaloshinskii-Moriya interaction, skyrmion Hall effect, Synthetic Antiferromagnets.

\section{INTRODUCTION}

$\mathrm{C}$ hiral magnetic textures, such as domain walls (DWs) [17] and skyrmions (Sks) [8-18], are nowadays the focus of active research as they offer great potential as information carriers for robust, high-density, and energy-efficient spintronic devices [19]. These magnetic patterns are formed due to the interfacial Dzyaloshinskii-Moriya interaction (DMI) [20-21] in asymmetric multilayers with high perpendicular magnetocristalline anisotropy (PMA), which consist in a thin ferromagnetic (FM) strip sandwiched between a heavy metal (HM) and an oxide (Ox), or between two different heavy metals. Both DWs and Sks can be efficiently driven along the FM strip by application of electrical current along the HM as due to the spin Hall effect (SHE) [22,24].

Recent experiments have shown that Néel-like Sks can be stabilized at room temperature and zero magnetic field in ultrathin FM/HM/Ox films, which makes their use appealing for the study of skyrmion structure and dynamics $[14,15,16,18]$. The magnetization of these chiral Néel Sks points radially $\left(\vec{u}_{\rho}\right)$ in the transition region between the inner and the outer parts, and its size depends on the composition and the thickness of the ferromagnetic layer. Néel-like Sks can be driven due to the SHE by injecting short current pulses with speeds exceeding $100 \mathrm{~m} / \mathrm{s}$ [14]. These observations promise an industrial integration. Nevertheless, the so-called "skyrmion Hall effect" [25,26] limits its movement along FM straight strips, as it imposes a transverse deviation from the center of the strip, and therefore, eventually results in their annihilation at the edge of the tracks. This reduces the maximum current that can be applied [17] and consequently the performance of Sky-based spintronic devices.

An alternative to the single FM layer stacks (HM/FM/Ox) has been recently proposed by Yang et al. in Ref. [27]. It consists in two FM layers separated by a Ru spacer (S) which, depending on its thickness, generates an antiferromagnetic coupling between the FM layers. In these synthetic antiferromagnetic multilayers (SAFs), the chiral Néel DWs in the lower and in the Upper FM layers are coupled each other, and they are efficiently driven by trains of current pulses, with velocities of $\sim 750 \mathrm{~m} / \mathrm{s}$ for current densities of $J \sim 2 \mathrm{TA} / \mathrm{m}^{2}$. This observation constitutes a significantly enhance of the efficiency with respect to the single-FM-layer stacks. The coupled DWs in these SAF stacks move rigidly, without rotation of their plane with respect to the current direction (no DW tilting) [27,28,29]. Moreover, adjacent DWs (up-down and down-up DWs) move with different velocities when they are forced to travel along curved parts in single-FM-layer stack, whereas they move with the same velocity and without tilting in the SAF track $[28,29]$. These observations suggest that trains of DWs can be driven along these systems with high-density packing of the encoded information.

Based on these results for DWs, Zhang and co-workers [30] have recently studied the current-driven skyrmion dynamics along straight SAF stacks from a theoretical and numerical point of view. Their modeling indicated that the two skyrmions in the Lower and in the Upper FM layers move together in a coupled manner, similarly to the coupled DWs experimentally analyzed by Yang et al. in [27]. The velocity of the antiferromagnetically coupled skyrmion increases linearly with the applied current without any significant "skyrmion Hall effect" along straight tracks [30,31,32,33,34,35], which constitutes major step towards the realization of reliable skyrmion-based spintronics devices. However, the currentdriven dynamics of Néel skyrmions along curved tracks have not been analyzed yet, neither along single-FM layer stacks nor along SAFs, and this is precisely the aim of the present work.

Here, we theoretically study the skyrmions dynamics under current pulses along a perfect and realistic FM multilayers by means of micromagnetic simulations. Straight and curved tracks with a single-FM-layer and multilayers with two FM 
layers antiferromagnetically coupled (SAFs) are studied. The current-driven skyrmion dynamics (CDSD) is also analytically described in the framework of the Thiele model, which considers the skyrmion as a rigid object $[9,10,11,17]$. Our results show the drawbacks of single-FM layer stacks to develop 2D circuits, and how the synthetic antiferromagnetic architecture constitutes a promising platform for skyrmionbased racetrack memory and logic devices.

\section{MicromagnetiC MODEL}

The multilayers studied here are schematically depicted in Fig. 1. The single-FM-layer stack (HM/FM/Ox) is ahown in Fig. 1(a), and the stack with two FM layers separated by a spacer (LHM/LFM/D/UFM/UHM) is schematically depicted in Fig. 1(b).

(a) Single FM layer stack (HM/FM/Ox)
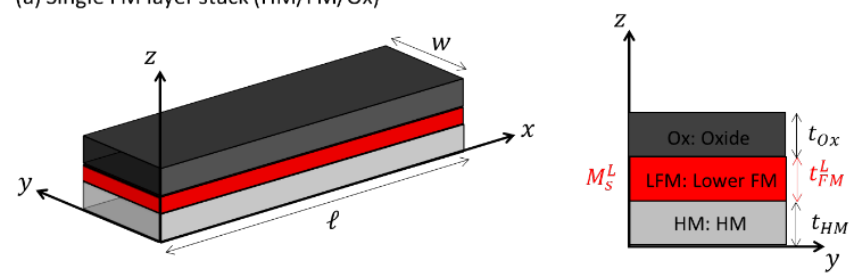

(b) Two FM layers heteroestructure (LHM/LFM/S/UFM/UHM)
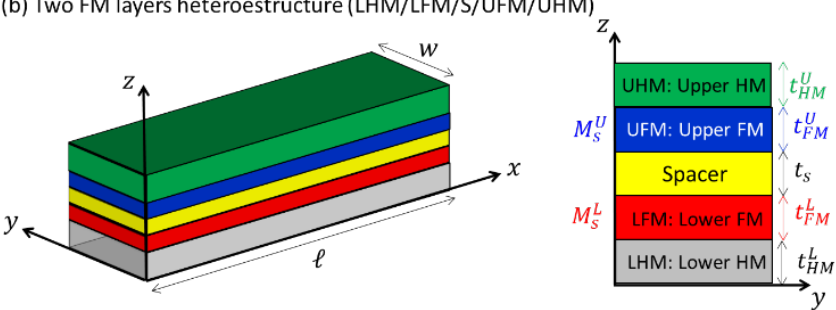

Fig. 1. Schematic view of the multilayers under study: (a) Single FMlayer stack $(\mathrm{HM} / \mathrm{FM} / \mathrm{Ox})$ and (b) Two FM layers heteroestructure (LHM/LFM/S/UFM/UHM). The definition of the acronyms is also given in the graphs. The relevant thicknesses for this study are marked on the figure, which are fixed to $t_{F M}^{L}=t_{S}=t_{F M}^{U}=1 \mathrm{~nm}$, except otherwise is indicated. The width of the strips is $w=192 \mathrm{~nm}$. Material parameters are given in the text.

Full micromagnetic $(\mu M)$ simulations have been performed by solving the Landau-Lifshitz-Gilbert equation augmented with the spin transfer torque $\left(\vec{\tau}_{S T}, \mathrm{STT}\right)$ and Slonczewski-like spin-orbit torque $\left(\vec{\tau}_{S O}, \mathrm{SOT}\right)$ due to the spin Hall torque [29,36,37]:

$$
\frac{d \vec{m}}{d t}=-\gamma_{0} \vec{m} \times\left(\vec{H}_{e f f}+\vec{H}_{t h}\right)+\alpha \vec{m} \times \frac{d \vec{m}}{d t}+\vec{\tau}_{S T}+\vec{\tau}_{S O}
$$

where $\gamma_{0}$ and $\alpha$ are the gyromagnetic ratio and the Gilbert damping constant respectively. $\vec{m}(\vec{r}, t) \equiv \vec{m}^{i}(\vec{r}, t)=\vec{M}^{i}(\vec{r}, t) /$ $M_{S}^{i}$ is the normalized local magnetization to its saturation value $\left(M_{s}^{i}\right)$, defined differently for each FM layer: $M_{s}^{i}$ where $i: L, U$ for the LFM and the UFM layers respectively. $\vec{H}_{\text {eff }}$ is the deterministic effective field, which includes not only the intralayer exchange and the uniaxial anisotropy, but also the interlayer exchange [38] and the magnetostatic interactions adequately weighed to account for the different saturation magnetizations. The interlayer exchange contribution $\left(\vec{H}_{e x}^{\text {inter }}\right)$ to the effective field $\vec{H}_{e f f}$, acting on each FM layer, is computed from the corresponding energy density $\left(\omega_{e x}^{i n t e r}=-\frac{J^{e x}}{t_{S}} \vec{m}^{L}\right.$. $\vec{m}^{U}$, where $J^{e x}$ is the interlayer exchange coupling parameter, $t_{S}$ is the thickness of the spacer between the LFM and the UFM layers, and $\vec{m}^{L}$ and $\vec{m}^{U}$ represent the normalized magnetization in the Lower and in the Upper layers respectively) as

$$
\vec{H}_{e x, i}^{\text {inter }}=-\frac{1}{\mu_{0} M_{s}^{i}} \frac{\delta \omega_{e x}^{\text {inter }}}{\delta \vec{m}^{i}}=\frac{J^{e x}}{\mu_{0} M_{s}^{j} t_{s}} \vec{m}^{j}
$$

where $i, j: L, U$. Ferromagnetic (FM) and antiferromagnetic (AF) coupling cases can be evaluated by a positive $J^{e x}$, and by a negative $J^{e x}$ respectively. Here we focus our attention to the AF coupling case, which is the most relevant in terms of efficiency.

The effective field in the LFM and in the UFM layers also includes the interfacial DMI at the LHM/LFM and UFM/UHM interfaces respectively. These interfacial DMI supports the formation of skyrmions with the same chirality and opposite polarity in both FM layers, that is, the DMI parameters $\left(D^{i}\right)$ are of opposite sign $\left(\operatorname{sign}\left(D^{L}\right)=-\operatorname{sign}\left(D^{U}\right)\right)$. The polarity of each skyrmion can be seen in Fig. 2. The rest of numerical details of other contributions to the effective field can be found elsewhere $[29,36] . \vec{H}_{t h}$ is the thermal field, included as a Gaussian-distributed random field $[39,40] . \vec{\tau}_{S O}$ represents the spin-orbit torque (SOT), which in the present work is acting on the two LFM and UFM layers. This torque is given by the Slonczewski-like term $\vec{\tau}_{S O}=-\gamma_{0} \vec{m} \times \vec{H}_{S L}$, where $\vec{H}_{S L}=$ $H_{S L}^{0} \vec{m} \times \vec{\sigma}$ is the Slonczewski-like effective field. Here, $\vec{\sigma}=$ $\vec{u}_{z} \times \vec{u}_{J}$ is the unit vector along the direction of the polarization of the spin current generated by the spin Hall effect (SHE) in the LHM, being orthogonal to both the direction of the electric current $\vec{u}_{J}$ and the vector $\vec{u}_{z}$ standing for the normal to the $\mathrm{HM} / \mathrm{LFM}$ interface. Finally, $H_{S L}^{0}=\hbar \theta_{S H}^{i} J_{H M} /\left(2 \mu_{0}|e| M_{S} t_{F M}\right)$ determines the strength of the SHE within each FM layer $(i=$ $\{U, L\})[2,4]$, where $\hbar$ is the Planck constant, $e$ is the electron charge, $\mu_{0}$ is the vacuum permeability, $\theta_{S H}^{i}$ is the spin Hall angle $(i=\{U, L\})$, and $J$ is the magnitude of the current density $\vec{J}_{H M}(t)=J_{H M}(t) \vec{u}_{J}$. For straight samples $\vec{u}_{J}=\vec{u}_{x}$, whereas for curved strips the direction and the local amplitude of current is previously computed by finite element method solvers [41]. On the other hand, Eq. (2) includes the spin transfer torques (STTs, $\vec{\tau}_{S T}$ ) due to the electrical current flowing across the FM layers $\left(\vec{J}_{F M}^{i}(t)=J_{F M}^{i}(t) \vec{u}_{J}\right.$, with $\left.i=\{U, L\}\right)$. This STTs [40] includes both adiabatic and non-adiabatic contributions: $\vec{\tau}_{S T}=b_{S T}^{i}\left(\vec{u}_{J}\right.$. $\nabla) \vec{m}-\xi_{i} b_{S T}^{i} \vec{m} \times\left(\vec{u}_{J} \cdot \nabla\right) \vec{m}$, where $b_{S T}^{i}=\frac{\mu_{B} P}{|e| M_{S}^{i}} J_{F M}^{i}$ is the STT coefficient, with $P$ the polarization factor and $J_{F M}^{i}$ the density current flowing directly throw the FM layer $i=\{U, L\} . \xi_{i}$ is the non-adiabatic coefficient [40].

Typical parameters have been considered in our simulations. Except where the contrary is indicated, $M_{s}$ values for the UFM and the LFM layers have been chosen respectively as $M_{s}^{L}=$ $1.0 \mathrm{MA} / \mathrm{m}$ and $M_{s}^{U}=1.0 \mathrm{MA} / \mathrm{m}$. The anisotropy constant, the intralayer exchange constant and the Gilbert damping are $K_{u}=$ 
$0.8 \mathrm{MJ} / \mathrm{m}^{3}, A=20 \mathrm{pJ} / \mathrm{m}$ and $\alpha=0.1$ for both FM layers. The interfacial DMI is $D^{L}=1.8 \mathrm{~mJ} / \mathrm{m}^{2}$ in the lower FM, and $D^{U}=$ $-1.8 \mathrm{~mJ} / \mathrm{m}^{2}$ in the upper FM layer. The spin Hall angle representing the degree of polarization of the vertical spin current acting on the LFM is $\theta_{S H}^{L}=-0.33$, whereas in the UFM is $\theta_{S H}^{U}=+0.33$. Note that in order to promote the coupledskyrmion dynamics along the same direction in the two FM layers of a SAF both the sign of the DMI parameter and the spin Hall angles have to be opposite: $\operatorname{sign}\left(D^{L}\right)=-\operatorname{sign}\left(D^{U}\right)$ and $\operatorname{sign}\left(\theta_{S H}^{L}\right)=-\operatorname{sign}\left(\theta_{S H}^{U}\right)$. These conditions can be obtained by choosing the HMs as Pt and W for the LHM and the UHM respectively. Different values of the interlayer exchange parameter $\left(J^{e x}\right)$ have been considered with magnitudes within the range $0 \leq\left|J^{e x}\right| \leq 0.5 \mathrm{~mJ} / \mathrm{m}^{2}$, but with $J^{e x}$ taking by default the value $J^{e x}=-0.5 \mathrm{~mJ} / \mathrm{m}^{2}$ for AF coupling.

For the study of single-FM-layer (HM/FM/Ox), the following parameters were adopted: $M_{S}=1.0 \mathrm{MA} / \mathrm{m}, K_{u}=$ $0.8 \mathrm{MJ} / \mathrm{m}^{3}, \quad A=20 \mathrm{pJ} / \mathrm{m}, \quad \alpha=0.1, \quad D=1.8 \mathrm{~mJ} / \mathrm{m}^{2}$ and $\theta_{S H}=-0.33$, which coincide exactly with the ones chosen for the LFM layer in the two FM layer case. The micromagnetic snapshots of the static equilibrium configuration of the skyrmions in the single FM layer and in the stack with two FM layers stacks are presented in Fig. 2(a) and (b) respectively. Fig. 2(c) and (d) depict the longitudinal and perpendicular magnetization components $\left(m_{x}(x)\right.$ and $\left.m_{z}(y)\right)$ along the central line $(y=0)$ of the ferromagnetic strips for the single FM layer and the SAF stacks respectively. As it is shown, the coupled consists of two mirror skyrmions: the skyrmion in the Lower FM layer of the SAF is identical to the one in the single FM stack, whereas the skyrmion in the Upper FM layer of the SAF has a reversed magnetization due to the antiferromagnetic coupling between these Lower and the Upper FM layers.

(a) Single FM layer stack

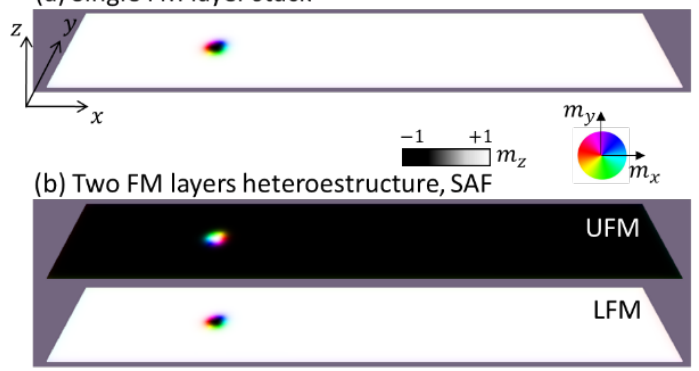

(c) Single FM laye

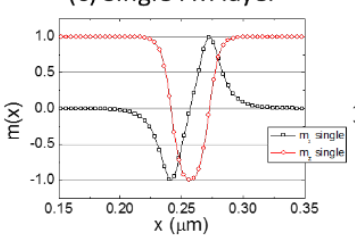

(b) SAF

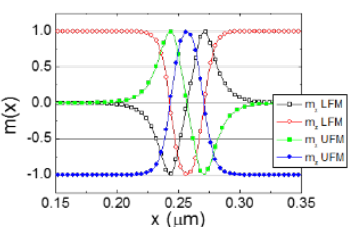

Fig. 2. Static skyrmion configurations in the single FM layer stack (a) and in the two FM layers heteroestructure (SAF). (b). The definition of the acronyms are also given in the graphs. (c) and (d) show the longitudinal and perpendicular magnetization components $\left(m_{x}(x)\right.$ and $\left.m_{z}(y)\right)$ along the central line $(y=0)$ of the ferromagnetic strips, for the single FM layer and the SAF stacks respectively. The width of the strips is $w=192 \mathrm{~nm}$. Material parameters are given in the text.
To evaluate the skyrmions dynamics, we assume that STT is negligible $\left(J_{F M}^{i}=0, b_{S T}^{i}=0\right)$, and that the electrical current flows only along the heavy metals of the two evaluated systems. Eq. (1) is solved using MuMax3 [42] which was adapted to include the Ruderman-Kittel-Kasuya-Yosida (RKKY) interaction [38] between non-adjacent FM layers separated by the spacer (S). The in-plane side of the computational cells is $\Delta x=\Delta y=1 \mathrm{~nm}$ and different thicknesses $\Delta z$, depending on the thickness of the FM layers, were considered. A homemade micromagnetic solver was also used to verify the validity of the obtained results. Except the contrary is said, the presented results were obtained at zero temperature. Simulations at room temperature were performed with a fixed time step $\Delta t=0.1 \mathrm{ps}$. Several tests were performed with reduced cell sizes and time steps to assess the numerical validity of the presented results.

Part of the simulations were carried out by considering perfect samples (Sec. III.a), without imperfections nor defects. However, other parts were computed under realistic conditions (see Sec. III.b). In order to take into account the effects of disorder due to imperfections and defects in a realistic way, we assume that the easy axis anisotropy direction is distributed among a length scale defined by a 'grain size'. The grains vary in size taking an average size of $10 \mathrm{~nm}$. The direction of the uniaxial anisotropy of each grain is mainly directed along the perpendicular direction ( $z$-axis) but with a small in-plane component which is randomly generated over the grains. The maximum percentage of the in-plane component of the uniaxial anisotropy unit vector is varied from $10 \%$ to $15 \%$. The presented results correspond to an in-plane maximum deviation from the out-of-plane direction of $12 \%$. Although other ways to account for imperfections and defects could be adopted, we selected this one based on previous studies, which properly describe other experimental observations [37].

\section{RESULTS AND DISCUSSION}

\section{III.a. Current-driven skyrmion dynamics along straight stacks}

Before describing the current-driven skyrmion dynamics along curved stacks, it is interesting to review the main features of the skyrmion motion along straight stacks. The transient micromagnetic snapshots in Fig. 3(a) show the temporal evolution of the skyrmion along a single FM layer stack under a current of $J=+0.02 \mathrm{TA} / \mathrm{m}^{2}$. The skyrmion is initially located at the center of the transversal direction of the FM layer, $Y(0)=0$. During the first nanoseconds $(t \lesssim 10 \mathrm{~ns})$, the skyrmion is essentially displaced along the transverse direction $Y(t)$, whereas its longitudinal position remains close to its initial value $(X(10 \mathrm{~ns}) \approx X(0))$. As the time elapses longer ( $t \gtrsim 10 \mathrm{~ns}), Y(t)$ reaches a terminal equilibrium value $Y_{e q}$, and the longitudinal coordinate $(X(t))$ starts to increase monotonously. This threshold value $Y_{e q}$ is due to the balance between the repulsive force from the edge and the gyromagnetic force, which deflects the skyrmions from its initial location in the center of the stack $(Y(0)=0)$. This is the so-called "skyrmion Hall effect". The transversal deflection increases with the current $J\left(Y_{e q} \propto J\right)$, and there is a critical value $J_{t h}$ 
above which the skyrmion is annihilated at the edge because the gyrotropic force becomes larger than the repulsive force from the edge. In the particular case of the materials and dimensions considered here, this threshold current is $J_{t h}=+0.04 \mathrm{TA} / \mathrm{m}^{2}$. Therefore, the maximum longitudinal velocity $\left(v_{x}\right)$ reached by the skyrmion is limited by the "skyrmion Hall effect" in single FM stacks. This terminal longitudinal velocity is $v_{x}$ is $\sim 24 \mathrm{~m} / \mathrm{s}$ for $J=+0.02 \mathrm{TA} / \mathrm{m}^{2}$, which is slightly below the annihilation threshold. The micromagnetic snapshots in Fig. 3(a) also indicate that the size of the skyrmion decreases as it is deflected along the transverse $y$ direction. This size reduction is due to the increasing of the magnetostatic compression of the edge.
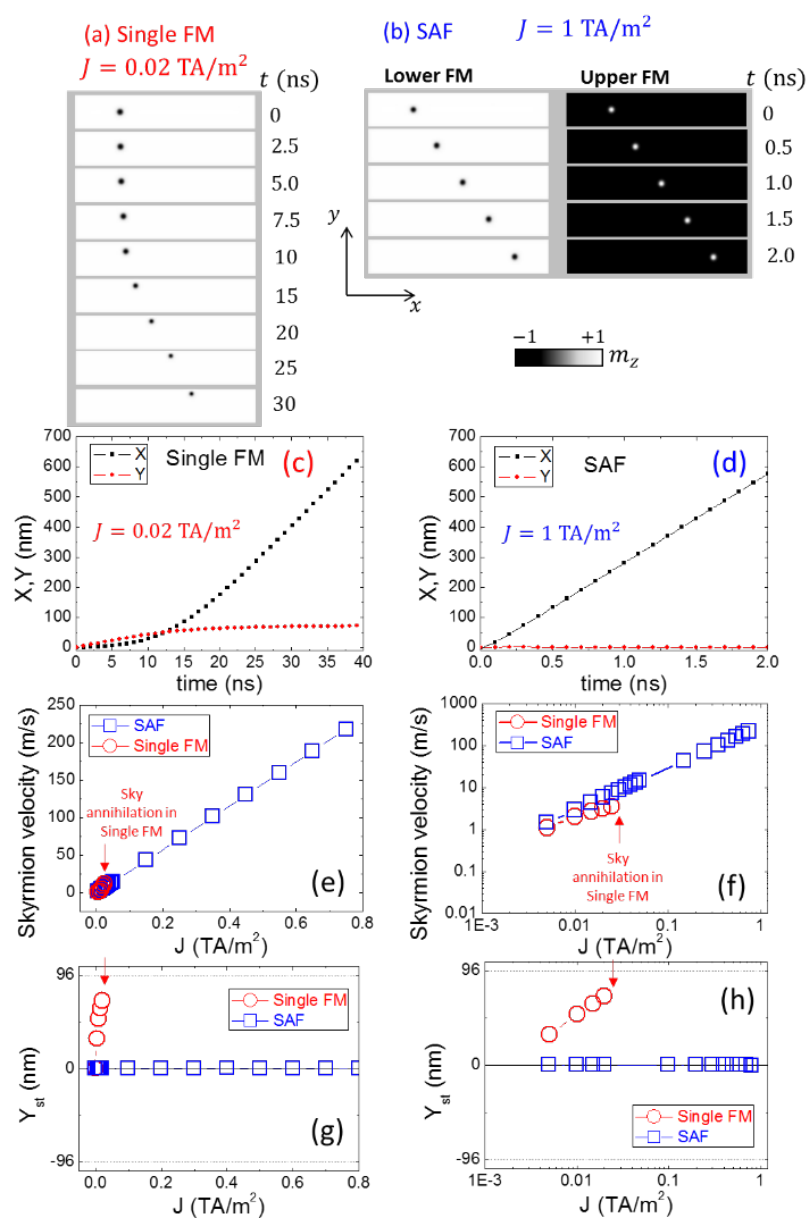

Fig. 3. Micromagnetic snapshots showing the temporal evolution of the skyrmion position along the FM layers in the following cases: (a) single-FMlayer, and (b) SAF coupling $\left(J^{e x}<0\right)$. UFM and LFM layers are simultaneously shown in case (b). The amplitude of the current pulse is $J=$ $+0.02 \mathrm{TA} / \mathrm{m}^{2}$ for the single-FM-layer case (a) and $J=+1 \mathrm{TA} / \mathrm{m}^{2}$ for the SAF case (b). Perfect samples and zero temperature are considered here. (c) and (d) show the temporal evolution of the skyrmion positions corresponding to the snapshots of (a) and (b). The comparison of the dependence of the terminal longitudinal skyrmion velocity $\left(v_{x}\right)$ as a function of $J$ for single FM and SAF cases is shown in (e) and (f) graphs. The terminal transverse deviation $\left(Y_{s t}\right)$ as a function of the current $(J)$ thru the HM/HMs is also shown in (g) and (h) graphs, which further supports the annihilation of the skyrmion in the single FM layer stack for relative low values of $J$, whereas the coupled skyrmion in the SAF moves without transverse deflection.

The situation is significantly different in stacks with two FM layer coupled antiferromagnetically $\left(J^{e x}<0\right)$ by a spacer (see
Fig. 1(b) and Fig. 2(b) and (d)). The temporal evolution of the coupled-skyrmion along this synthetically antiferromagnetic stack (SAF) is shown in Fig. 3(b) under a current of $J=$ $+1.0 \mathrm{TA} / \mathrm{m}^{2}$, which is $\sim 50$ times larger than the threshold value in the single FM stack of Fig. 3(a). The coupled-skyrmion consists on two mirror skyrmions within each of the two FM layers of the SAF (see Fig. 2(b)), and it is driven with high velocity without any significant deflection along the transverse direction (Fig. 3(b)). It is also clear that the size of the coupled skyrmion is preserved during its motion, which is again a consequence of the absence of skyrmion Hall effect in this SAF system. Besides of the high velocity and the absence of skyrmion Hall effect, it is also remarkable that the coupledskyrmion starts to move along the longitudinal direction without any inertia as soon as the current is applied (Fig. 3(b) and (d)). This is very different from the single FM layer case (Fig. 3(a) and (c)), where the longitudinal motion only reaches a constant longitudinal velocity once the transversal equilibrium threshold is reached after a several nanoseconds (Fig. 3(a) and (c)). See Ref. [17] for analytical expression of the characteristic time needed by the skyrmion to reach the steadystate regime with $v_{x, s t}$ constant in single FM layer case. Although it is not shown here, it was also verified that the coupled skyrmion in the SAF stops suddenly if the current is turned off, which also is indicative of the lack of inertial effects in these SAF systems. All these features, $\boldsymbol{i}$ ) the absence of the skyrmion Hall effect, ii) the high velocity and iii) the negligible inertia of the coupled-skyrmions in the SAF stacks, make them a very promising platform to develop skyrmion-based memory and logic devices.

The full micromagnetic results along the straight tracks presented in Fig. 3 can be analytically understood in the framework of the Thiele model [17], which describes the skyrmion as a rigid object. In a single-FM-layer with transverse cross section $w \times t$ ( $w$ : width, $t \equiv t_{F M}$ : thickness) the $2 \mathrm{D}$ skyrmion dynamics is given by:

$$
\vec{G} \times \vec{v}+\alpha \overleftrightarrow{D} \cdot \vec{v}=\vec{F}_{S H E}+\vec{F}_{C}
$$

where $\vec{v}=v_{x} \vec{u}_{x}+v_{y} \vec{u}_{y} \quad$ is the skyrmion velocity ( $v_{x}=$ $\left.d X / d t, \quad v_{y}=d Y / d t\right) . \quad \vec{G}=-4 \pi(p q) \frac{\mu_{0} M_{s}}{\gamma_{0}} t \vec{u}_{x} \quad$ is $\quad$ the gyrovector, where $p$ and $q$ represent the skyrmion polarity ( $p=$ $\pm 1)$ and topological charge $(q)$ respectively. $\overleftrightarrow{D}$ is the $2 \times 2$ diagonal dissipation tensor, with elements $D_{i i}=D \delta_{i i} \approx$ $-\frac{\mu_{0} M_{S}}{\gamma_{0}} t\left(\frac{\pi^{3} R_{S k}}{\Delta}\right) . \quad \vec{F}_{C}$ is the restoring force due to the confinement (i.e. imposed by the strip edges), which in a first approximation can be described as $\vec{F}_{C}=-k Y \vec{u}_{y}$ with $k$ being an elastic constant, which can be estimated from a single micromagnetic simulation [17]. $\vec{F}_{S H E}$ is the driving force due to the SHE, which is given by $\vec{F}_{S H E} \approx-\frac{\hbar \theta_{S H J}}{2 e} \pi^{2} \eta R_{S k} \vec{u}_{x}=$ $F_{S H E} \vec{u}_{x}$. Eq. (2) describes the motion of a rigid skyrmion with a characteristic size of given by $\eta R_{S k}$. The analytical solution of Eq. (2) gives the temporal evolution of the longitudinal $(X=$ $X(t))$ and transverse $(X=X(t))$ skyrmion coordinates, and it 
can be consulted in [17]. The steady-state or terminal longitudinal velocity $v_{x, s t}$ and the terminal transverse displacement $Y_{s t}$ can be deduced from that solution. The resulting expression are $v_{x, s t}=\frac{F_{S H E}}{\alpha D}$ and $Y_{s t}=\frac{G F_{S H E}}{\alpha D k}$. Note that the terminal transverse displacement $Y_{s t}$ is proportional to the modulus of the gyrovector $G$, which depends on the topological charge $(q)$. For example, under a current of $J=0.02 \mathrm{TA} / \mathrm{m}^{2}$, the micromagnetic results for the terminal values are $v_{x, s t} \approx$ $24 \mathrm{~m} / \mathrm{s}$ and $Y_{s t} \approx 72 \mathrm{~nm}$. From these results we deduce $k=$ $0.24 \times 10^{-4} \mathrm{~N} / \mathrm{m}$ using $R_{S k}=15 \mathrm{~nm}, \eta=1.2$ and $\Delta=$ $16 \mathrm{~nm}$. It was verified that with these inputs, the analytical model provides a good quantitative agreement with the micromagnetic results of Fig. 3(c) and (e).

On the other hand, the Thiele model (Eq. (2)) can be also adapted to explain the micromagnetic results presented for SAF stacks, as the two skyrmions can be described as "single coupled skyrmion". The two skyrmions in the two ferromagnetic layers have opposite topological charge $\left(q^{L}=\right.$ $-q^{U}$ ), and consequently, the total topological charge of the antiferromagnetically coupled skyrmion is null, $q_{\text {total }}=q^{L}+$ $q^{U}=0$. Therefore, the gyrovector $\vec{G}$ of the coupled skyrmion is also zero $(\vec{G}=0)$, and consequently, the transverse deflection is identically zero $\left(Y_{s t}=\frac{G F_{S H E}}{\alpha D k}=0\right)$, which explains the absence of skyrmion Hall effect in these SAF systems (see Fig. $3(d))$. Moreover, as the coupled skyrmion is not deflected along the transverse direction, higher current densities can be injected, and larger velocities are achieved as compared to the single FM layer case. Note that the longitudinal velocity of the coupled skyrmion can be also described be the same analytical expression, $v_{x, s t} \approx \frac{F_{S H E}}{\alpha D}$, which explains the linear dependence of $v_{x, s t}$ on $J$, as shown by the micromagnetic results of Fig. 3(e) and (f)

\section{III.b. Current-driven dynamics along curved stacks}

Previous section stated the advantages of the current-driven skyrmion dynamics in a straight SAF stack over the single FM layer case. However, the design of skyrmion-based devices will also require to analyze and to control the skyrmion dynamics along 2D tracks, which will consist on straight and curved parts. Note that in curved tracks, where the heavy metals are needed to be patterned with the same shape than the FM layers, the electrical current $\vec{J}=\vec{J}(\vec{r})$ will be non-uniform, and therefore, it is needed to evaluate the influence of the non-uniform current on the skyrmion dynamics. In order to do it, we have considered both single FM layer and SAF stacks with the in-plane (xy) shape shown in Fig. 4(a), which contains straight and curved parts. The definitions of the geometrical parameters and the values considered in the present analysis are shown in Fig. 4(a) and in its caption. The strip width is $w_{s}=w$ and the thickness of the different layers are the same as in the former Sec. III.a. The same material parameters are also considered here. See Appendix for a detailed explanation of the non-uniform current density in curved strips.

The current distribution $\vec{J}(\vec{r})$ in the HMs is shown in Fig. 4(b), which clearly indicates a radial dependence: the current density $\vec{J}(\vec{r})$ depicts an inversely linear dependence on the radius when is forced to flow over semicircular arcs. Notice the values of the magnitude of the current $|\vec{J}(\vec{r})|$ in the curved parts (vertical color scale in Fig. 4(b)), which are normalized to its uniform value $\left(J_{u}\right)$ along the straight part. The current becomes significantly higher in the inner part of the curve $\left(|\vec{J}(\vec{r})| / J_{u}>\right.$ 1 ), and its value decreases towards the outer part, where its magnitude becomes smaller than in the straight part $(|\vec{J}(\vec{r})| /$ $\left.J_{u}<1\right)$. These results suggest that the skyrmion dynamics could be significantly modified when it is forced to pass along curved parts, where the driving force due to the spin Hall effect is proportional to the electrical current along the HMs. The spatial distribution of the current was computed with COMSOL [41] and taken into account to evaluate the current driven skyrmion motion by numerically solving Eq. (1).

Before comparing single FM layer and SAF results it is interesting to describe the limitations of the single FM layer stack to drive series of skyrmions along samples with curved parts. Fig. 5 shows the dynamics of two skyrmions under a current of $J_{u}=J=0.01 \mathrm{TA} / \mathrm{m}^{2}$, which indicates the value at the straight parts, and it was chosen to be below the annihilation threshold. The non-uniform distribution of the current is taken into account (Fig. 4b). The skyrmions are initially placed at the central line of the stack along the longitudinal direction, that is, $X_{t}(0)=X_{m}(0)=0$, where the here $t$ and $m$ represent the top and the middle skyrmions. As it is shown, the top skyrmion is placed at the top straight part and the other one in the middle central straight part. The top skyrmion is driven to cross the topright curve in a clockwise direction $(\mathrm{CW})$, whereas the middle ones is forced to pass over the left-bottom curve in a counterclockwise direction $(\mathrm{CCW})$. The snapshots evidence a different velocity along the curved parts, which is a direct consequence of the skyrmion Hall effect and of the spatial distribution of the current. Indeed, the top skyrmion enters in the $\mathrm{CW}$ curve close to the outer radius $\left(r_{o}\right)$, where the local current density is smaller than in the straight part $\left(|\vec{J}(\vec{r})| / J_{u}<\right.$ 1 ), and therefore, it linear velocity is reduced with respect to the one in the straight part. On the other hand, the skyrmion initially placed at the middle enters in the CCW curve close to the inner radius $\left(r_{i}\right)$, where the local current is larger $\left(|\vec{J}(\vec{r})| / J_{u}>1\right)$, and therefore its linear velocity is larger than in the straight parts. It is also clear that the radial distance covered by the top skyrmion in its $\mathrm{CW}$ turn is larger than the one covered by the middle one in its CCW. These observations clearly show that the relative distance between the skyrmions is significantly modified as they are driven along a single FM layer stack with curved parts. Consequently, sigle FM layer stacks are not good platforms to develop skyrmion-based racetracks. Although the presented result were obtained under static currents, we have also verified that the mentioned limitations remain when the skyrmions are driven by current pulses: the skyrmion may not be annihilated if the current pulses are properly chosen, but the relative distance between skyrmions will change as they are moved 
along $\mathrm{CW}$ and $\mathrm{CCW}$ turns.
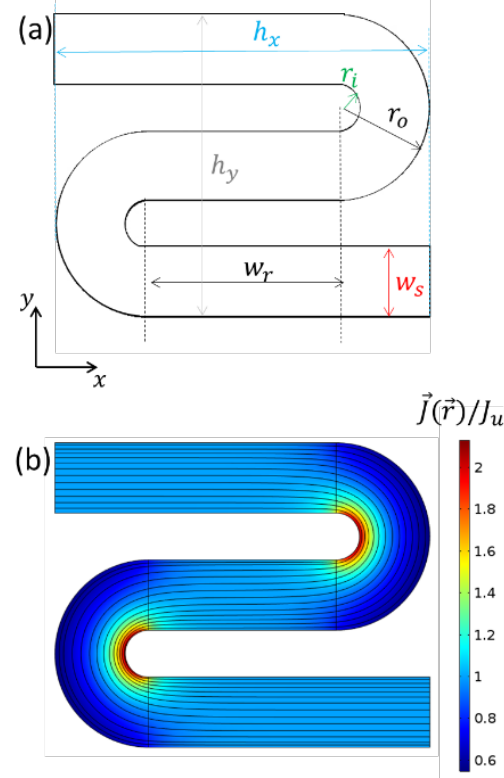

Fig. 4. In-plane ( $x y$-plane) geometry used to evaluate the current-driven Skyrmion dynamics along tracks with straight and curved parts. It contains three straight sections connected by two round-shaped sections. (a) The values of the geometrical parameters defined therein are: $w_{r}=512 \mathrm{~nm}, r_{i}=64 \mathrm{~nm}$, $r_{o}=256 \mathrm{~nm}, w_{s}=r_{o}-r_{i}, h_{x}=2 r_{o}+w_{r}$, and $h_{y}=2 r_{o}+2 r_{i}+w_{s}$. (b) Spatial distribution of the normalized current density $\left(\vec{J}(\vec{r}) / J_{u}\right)$. Color indicates the current density $(\vec{J}(\vec{r}))$ normalized to the value in the straight part $\left(J_{u}\right)$, where the current is uniform $J_{u}$. Lines only represent the local direction of the $\vec{J}(\vec{r})$.

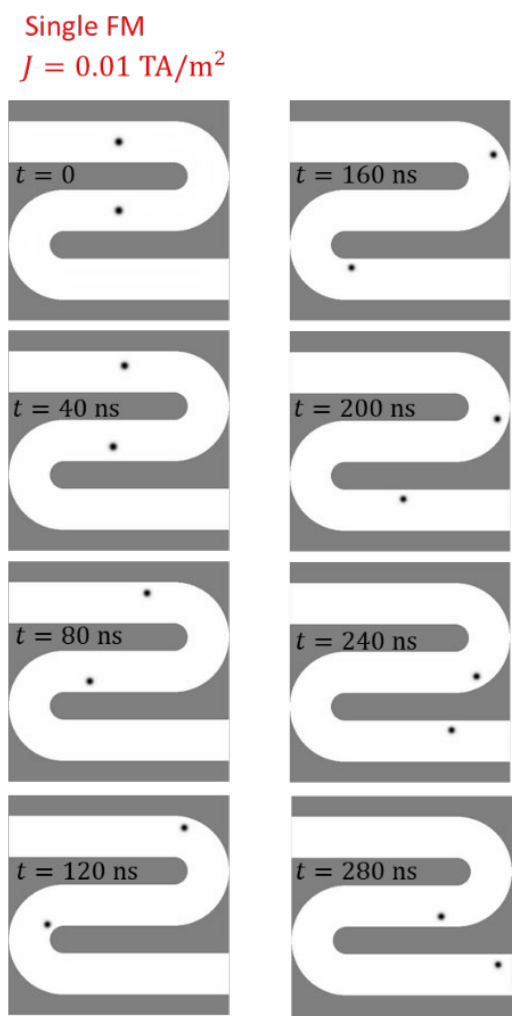

Fig. 5. Transient snapshots of the current-driven dynamics along a single FM layer curved track. The applied current is $J=0.01 \mathrm{TA} / \mathrm{m}^{2}$. Two skyrmions are driven by the same current. As they cross the curved parts, its relative distance changes as discussed in the text.
Once stablished the drawbacks of the single FM layer stack to drive skyrmions along samples with curved parts, we now compare the dynamics to the SAF stack case. To do it, realistic conditions have been considered, which include defects in the form of grains (see details at the end of Sec. II) and thermal effects at room temperature $(T=300 \mathrm{~K})$. As in Sec. III.a, two cases are considered here: a single-FM-layer stack (Fig. 6(a)) and a multilayer with two antiferromagnetically-coupled FM layers (Fig. 6(b), SAF).

In the single FM layer case, the skyrmion is initially driven from the left to the right along the top horizontal branch (Fig. 6(a)). In this horizontal part the current is uniform and the skyrmion depicts the mentioned "skyrmion Hall effect" but without annihilation before reaching the first right handed curve. Note that the skyrmion is not annihilated for this current because the short horizontal length of the top straight part and also due to the presence of defects. When the skyrmion enters in the first curve towards the right $(\mathrm{CW})$, the current becomes non-uniform, and its magnitude is smaller than in the straight part (see Fig. 4(b)) in the outer edge where the skyrmion is close. As the skyrmion is moving close to this outer part, its linear velocity is slightly smaller than in the straight horizontal. Therefore, it completes the first clockwise $180^{\circ}$ turn without annihilation, reaches the central horizontal branch, and moves up to reaching the second $180^{\circ}$ counter-clockwise turn. The skyrmion annihilates as soon as it enters in this second curve (left turn, CCW) (Fig. 6(a) at $t=225 \mathrm{ns)} \mathrm{because} \mathrm{the} \mathrm{high}$ current density (and consequently the high spin Hall force) in the inner part of this turn.

The dynamics of the coupled-skyrmion along curved tracks is completely different. Analogously to the straight case discussed in Fig. 3(b) where the current is uniform, the skyrmion is driven with high velocity along the middle of the track (Fig. 6(b)) even if the current is non-uniform as due to the curved path. Therefore, these realistic simulations, where disorder and thermal effects are taken into account along with the non-uniform current distribution, clearly point out the advantages of the SAF stacks as promising platforms to develop skyrmion-based devices.

\section{CONCLUSION}

The current-driven skyrmion dynamics have been theoretically analyzed different stacks with straight and curved parts. The analysis of the stacks with curved parts is relevant for future development of skyrmion 2D devices. In multilayers with a single FM layer, the skyrmion trajectory is deflected due to the skyrmion Hall effect, which is a transverse deviation with respect to the current direction in the heavy metal under the ferromagnetic layer. The terminal deviation increases with the current, and there is a threshold current value above which skyrmion is annihilated at the edge of the strip. Therefore, the maximum value of the skyrmion longitudinal velocity is limited by this skyrmion Hall effect. For driving currents well below this threshold, skyrmion can travel also along curved parts without annihilation. However, due to the non-uniform distribution of the current in the curved parts, the skyrmion 
velocity depends on the local radius of its curved trajectory, and this velocity is different from the velocity along the straight part. We have shown that the velocity is different for $\mathrm{CW}$ than $\mathrm{CCW}$ curves in a single FM layer stack, which is detrimental for racetrack applications. Indeed, for high currents but below the threshold value along the straight part, the skyrmion enters in the curved part at a certain distance to the close edge of the strip. If the skyrmion is moving close to the outer radius of the curved path its linear velocity decreases because the current density decreases with respect to its uniform value along the straight part. Therefore, the skyrmion can complete the turn without annihilation. On the contrary, if the skyrmion is forced to turn close to the inner radius of the local curved part (where the local current density is larger than its uniform value), its linear velocity increase accomplished by a larger deviation along the radial direction, which eventually results in the skyrmion annihilation. If the current sufficiently small to avoid annihilation, the difference velocity along $\mathrm{CW}$ and $\mathrm{CCW}$ curves modifies the relative distance between series of skyrmions, which is also detrimental for racetrack applications.
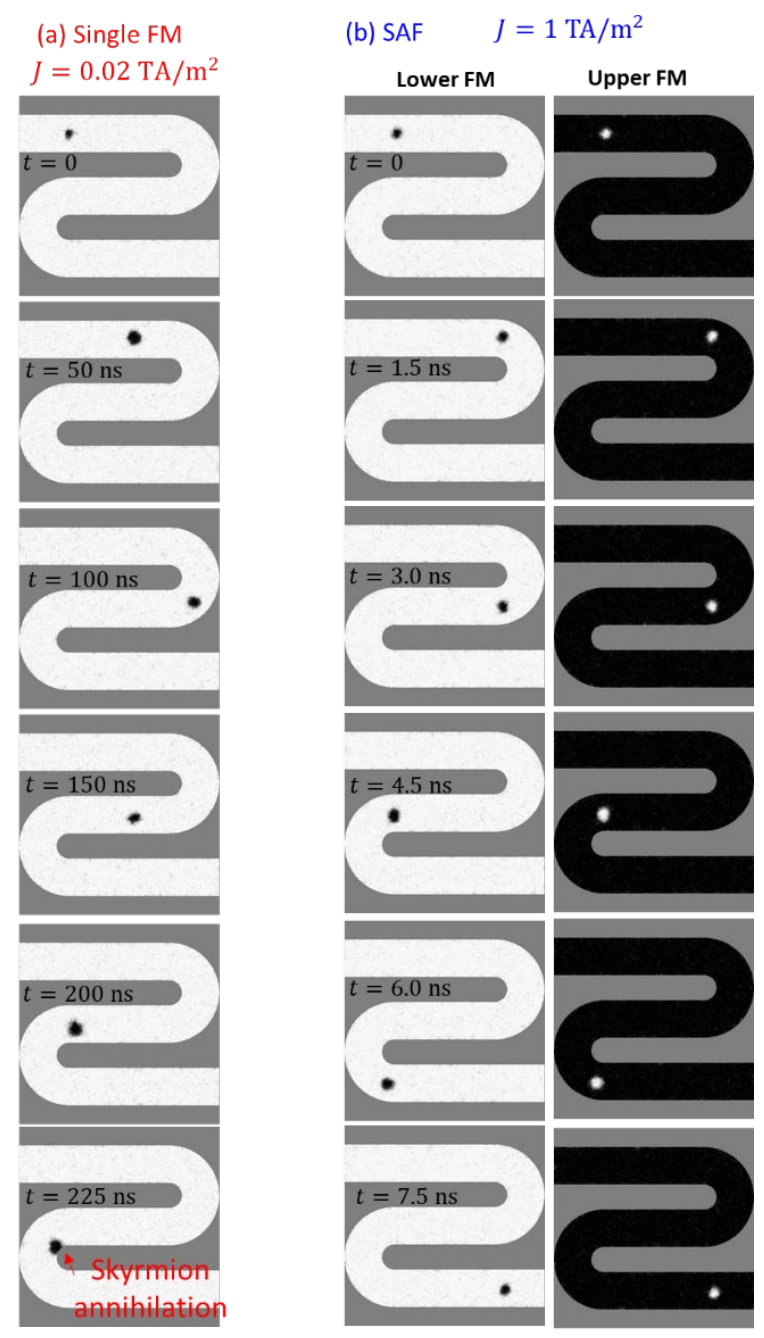

Fig. 6. (a) Transient snapshots of the current-driven Skyrmions dynamics along a single FM layer curved track. The applied current is $J=0.02 \mathrm{TA} / \mathrm{m}^{2}$, and each snapshot is shown after each $\Delta t=25 \mathrm{~ns}$. (b) Transient snapshots of the current-driven Skyrmions dynamics along a curved track consisting of two ferromagnetic layers with AFM coupling. The applied current is $J=1 \mathrm{TA} / \mathrm{m}^{2}$, and each snapshot is shown after each $\Delta t=1.5 \mathrm{~ns}$. Realistic conditions, with disorder (as described in Sec. II) and thermal fluctuations at room temperature, are considered here.

We have also evaluated the current-driven skyrmion dynamics in multilayers where two ferromagnetic layers are separated by a spacer, which generates an antiferromagnetic coupling between the ferromagnetic layers. In these synthetic antiferromagnetic stacks, the two skyrmions within each ferromagnetic layer moved coupled each other if the antiferromagnetic coupling is large enough without. As the coupled skyrmion in these systems does not depict skyrmion Hall effect, it is possible to apply much larger current, and consequently the coupled skyrmion reaches much higher velocities than in the single ferromagnetic layer counterparts. These advantages are also observed in samples with curved parts, where the coupled skyrmion also depicts a high velocity motion along the central path. Our results point out the drawbacks of the single ferromagnetic layer stack to develop skyrmion-based racetrack spintronic devices, and at the same time, the corresponding advantages of the synthetic antiferromagnetic systems to design such skyrmion-based devices.

\section{APPENDIX}

When a potential difference $(V)$ is applied between the two ends of a straight conducting strip with length $\ell$ along the longitudinal $x$-axis, the resulting electric field $(\vec{E})$ and the density current $(\vec{J}=\gamma \vec{E}$, with $\gamma$ being the electrical conductivity) are both uniform along the strip cross section ( $w \times t$, with $w$ and $t$ being the width and the thickness of the strip, along $y$ and $z$-axis respectively), that is, $\vec{E}=\frac{V}{\ell} \vec{u}_{x} \neq f(\vec{r})$ and $\vec{J}=\gamma \frac{V}{\ell} \vec{u}_{x} \neq f(\vec{r})$, where $\vec{r}$ is position vector. In this case, $\vec{J}$ can be also expressed as $\vec{J}=J_{u} \vec{u}_{x}$, where $J_{u}=I /(w t)$ is the uniform current density, with $I$ being the electrical current thru the strip. However, if the conducting strip is curved as shown in Fig. 6, the electric field and the corresponding current density are not uniform (that is, $\vec{E}=\vec{E}(\vec{r})$ and $\vec{J}=\vec{J}(\vec{r})$ ). Indeed, they have larger amplitude $(|\vec{E}|,|\vec{J}|)$ in locations close to the inner radius of the curve, and smaller close to the outer radius. When applying a potential difference $(V)$ between the two ends of a curved conductive strip (with thickness $t$, inner $r_{i}$ and outer $r_{i}$ radios, and conductivity $\gamma$ ), the current distribution $\vec{J}$ can be calculated analytically from the Ohm's law: $\vec{J}(\vec{r})=\gamma \vec{E}(\vec{r})=$ $\gamma \frac{V}{\pi r} \vec{u}_{\phi}$, where $r$ is the radius with respect to the center of the curve, and $\vec{u}_{\phi}$ is the unit vector along the azimuthal direction, with respect to the $x$-axis. Therefore, the amplitude of the density current $|\vec{J}|=J(r)$, depends on $r$, as $J(r) \propto \frac{1}{r}$, a fact which can be clearly observed in Fig. 3(b) along the radial direction within the two curves. A simply calculation allows us to express the $\vec{J}(\vec{r})$ along the curved strip in terms of the uniform current density of the straight strips $\left(J_{u}\right)$, as 


$$
J(r)=\frac{J_{u} w}{r \ln \left(1+\frac{w}{r_{i}}\right)}
$$

Eq. (3) is represented in Fig. 6(b) for a curved strip with the same dimensions as the curves of the system evaluated in Fig. 4: $r_{i}=64 \mathrm{~nm}$ and $r_{o}=256 \mathrm{~nm}$, which is in excellent quantitative agreement except for positions close to the connection with the straight parts. Indeed, in our system (see Fig. 4), which has straight and curved parts, there is not analytical solution at the connection of the straight part and the curved one. For this reason, we solved the Laplace eq. for the electrical potential numerically using a finite element solver, COMSOL [41], which solves the Laplace eq. $\nabla^{2} \psi=0$, with $\psi$ being the electric potential, with the Neumann boundary conditions which impose the potential at the ends of the conducting paths, the heavy metals. From the spatial dependence of the electric potential $\psi=\psi(\vec{r})$, the current distribution is obtained from Ohm's law as: $\vec{J}(\vec{r})=\gamma \vec{E}(\vec{r})$, where $\vec{E}(\vec{r})=-\nabla \psi(\vec{r})$ is the gradient of the electric potential. The direction of $\vec{J}(\vec{r})$ and the spatial dependence of its amplitude $(|\vec{J}|=J(r))$ are both shown in Fig. 4(b).

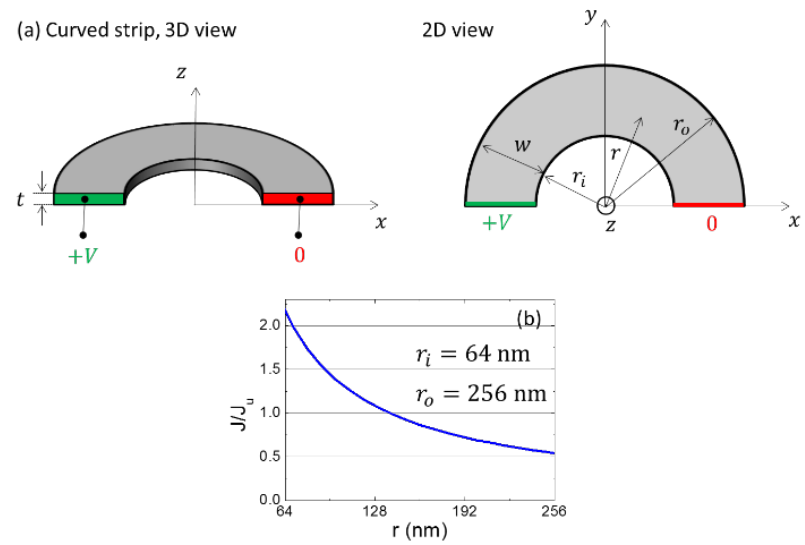

Fig. 7. (a) Geometry of a curved strip where the current distribution can be analytical calculated as described in the text. The geometrical parameters and the planes where the potential difference is applied, are defined in the image. (b) Normalized current density as a function of $r$ for a curved strip with $r_{i}=$ $64 \mathrm{~nm}$ and $r_{o}=256 \mathrm{~nm}$, as given by Eq. (3).

\section{ACKNOWLEDGMENT}

This work was supported in part by Spanish Governmentunder Project MAT2014-52477-C5-4-P, Project MAT2017-87072C4-1-P, and Project MAT2017-90771-REDT and in part by the Junta de Castilla y Leon under Project SA090U16 and Project SA299P18.

\section{REFERENCES}

[1] A. Thiaville, S. Rohart, É. Jué, V. Cros, and A. Fert, "Dynamics of Dzyaloshinskii domain walls in ultrathin magnetic films" Europhys. Lett. 100, 57002 (2012)

[2] S. Emori, U. Bauer, S.-M. Ahn, E. Martinez, and G. S. D. Beach, "Currentdriven dynamics of chiral ferromagnetic domain walls" Nature Mater. 12, 611-616 (2013).

[3] K.-S. Ryu, L. Thomas, S.-H. Yang, and S. Parkin, "Chiral spin torque at magnetic domain walls" Nature Nanotech. 8, 527-533 (2013).

[4] S. Emori et al., "Spin Hall torque magnetometry of Dzyaloshinskii domain walls" Phys. Rev. B 90, 184427 (2014).
[5] J. Torrejon, J. Kim, J. Sinha, S. Mitani, M. Hayashi, M. Yamanouchi, and $\mathrm{H}$. Ohno, "Interface control of the magnetic chirality in $\mathrm{CoFeB} / \mathrm{MgO}$ heterostructures with heavy-metal underlayers" Nat. Commun. 5, 4655 (2014).

[6] R. P. Del Real, V. Raposo, E. Martinez, and M. Hayashi, "Current-Induced Generation and Synchronous Motion of Highly Packed Coupled Chiral Domain Walls" Nano Lett. 17, 1814 (2017).

[7] O. Alejos, V. Raposo, L. Sanchez-Tejerina, and E. Martinez, "Efficient and controlled domain wall nucleation for magnetic shift registers" Sci. Rep. 7, 11909 (2017).

[8] A. Fert, V. Cros, and J. Sampaio, "Skyrmions on the track" Nat. Nanotechnol. 8, 152 (2013).

[9] J. Sampaio, V. Cros, S. Rohart, A. Thiaville, and A. Fert, "Nucleation, stability and current-induced motion of isolated magnetic skyrmions in nanostructures" Nat. Nanotechnol. 8, 839 (2013).

[10] J. Iwasaki, M. Mochizuki, and N. Nagaosa, "Universal current-velocity relation of skyrmion motion in chiral magnets" Nat. Commun. 4, 1463 (2013).

[11] J. Iwasaki, M. Mochizuki, and N. Nagaosa, "Current-induced skyrmion dynamics in constricted geometries" Nat. Nanotechnol. 8, 742 (2013).

[12] R. Tomasello, E. Martinez, R. Zivieri, L. Torres, M. Carpentieri, and G. Finocchio, "A strategy for the design of skyrmion racetrack memories" Sci. Rep. 4, 6784 (2014).

[13] W. Jiang, P. Upadhyaya, W. Zhang, G. Yu, M. B. Jungfleisch, F. Y. Fradin, J. E. Pearson, Y. Tserkovnyak, K. L. Wang, O. Heinonen, S. G. E. te Velthuis, and A. Hoffmann, "Blowing magnetic skyrmion bubbles" Science 349, 283 (2015).

[14] S.Woo, K. Litzius, B. Kruger, M.-Y. Im, L. Caretta, K. Richter, M. Mann, A. Krone, R. M. Reeve, M. Weigand, P. Agrawal, I. Lemesh, M.-A. Mawass, P. Fischer, M. Klaui, and G. S. D. Beach, "Observation of roomtemperature magnetic skyrmions and their current-driven dynamics in ultrathin metallic ferromagnets" Nat. Mater. 15, 501 (2016).

[15] C. Moreau-Luchaire, C. Moutafis, N. Reyren, J. Sampaio, C. A. F. Vaz, N. Van Horne, K. Bouzehouane, K. Garcia, C. Deranlot, P.Warnicke, P.Wohlh"uter, J.M. George, M.Weigand, J. Raabe, V. Cros, and A. Fert, "Additive interfacial chiral interaction in multilayers for stabilization of small individual skyrmions at room temperature" Nat. Nanotechnol. 11, 444 (2016).

[16] O. Boulle, J. Vogel, H. Yang, S. Pizzini, D. de Souza Chaves, A. Locatelli, T. Onur Mentes, A. Sala, L. D. Buda-Prejbeanu, O. Klein, M. Belmeguenai, Y. Roussigné, A. Stashkevich, S. M. Chérif, L. Aballe, M. Foerster, M. Chshiev, S. Auffret, I. M. Miron, and G. Gaudin, "Roomtemperature chiral magnetic skyrmions in ultrathin magnetic nanostructures" Nat. Nanotechnol. 11, 449 (2016).

[17] V. Raposo, R. F. Luis Martinez, and E. Martinez, "Current-driven skyrmion motion along disordered magnetic tracks" AIP Advances 7, 056017 (2017)

[18] W. Legrand et al "Room temperature current-induce generation and motion of sub-100nm skyrmions" Nano. Lett. 17, 2103-2712 (2017).

[19] S. S. P. Parkin, M. Hayashi, and L. Thomas, "Magnetic Domain-Wall Racetrack Memory" Science 320, 190 (2008).

[20] A. Dzyaloshinsky, "A thermodynamic theory of 'weak' ferromagnetism of antiferromagnetics" J. Phys. Chem. Solids 4, 241-255 (1958).

[21] T. Moriya, "Anisotropic superexchange interaction and weak ferromagnetism" Phys. Rev. 120, 91-98 (1960).

[22] L. Liu, T. Moriyama, D. C. Ralph, and R. A. Buhrman, "Spin-Torque Ferromagnetic Resonance Induced by the Spin Hall Effect" Phys. Rev. Lett. 106, 036601 (2011).

[23] L. Liu, C.-F. Pai, Y. Li, H. W. Tseng, D. C. Ralph, and R. A. Buhrman, Science 336, 555 (2012).

[24] J. Sinova, S. O. Valenzuela, J. Wunderlich, C. H. Back, T. Jungwirth, "Spin Hall effects", Rev. Mod. Phys. 87, 1213-1260 (2015).

[25] W. Jiang et al "Direct observation of the skyrmion Hall effect" Nat. Phys. 13, 162-169 (2017)

[26] K. Litzius, I. Lemesh, B. Krüger, P. Bassirian, L. Caretta, K. Richter, F. Büttner, K. Sato, O.A. Tretiakov, J. Förster, R.M. Reeve, M. Weigand, I. Bykova, H. Stoll, G. Schütz, G.S.D. Beach, and M. Kläui, "Skyrmion Hall effect revealed by direct time-resolved X-ray microscopy" Nature Physics 13, 170 (2017).

[27] S.-H. Yang, K.-S. Ryu, and S. Parkin, "Domain-wall velocities of up to $750 \mathrm{~m} s-1$ driven by exchange-coupling torque in synthetic antiferromagnets", Nat. Nanotechnol. 10, 221 (2015).

[28] C. Garg, S. Yang, T. Phung, A. Pushp, and S.S.P. Parkin, "Dramatic influence of curvature of nanowire on chiral domain wall velocity" Sci. Adv. 3, e1602804 (2017). 
[29] O. Alejos, V. Raposo, L. Sanchez-Tejerina, R. Tomasello, G. Finocchio, and E Martinez, "Current-driven domain wall dynamics in ferromagnetic layers synthetically exchange-coupled by a spacer: A micromagnetic study", J. Appl. Phys. 123, 013901 (2018).

[30] X. Zhang, Y. Zhou, and M. Ezawa, "Magnetic bilayer-skyrmions without skyrmion Hall effect" Nat. Commun. 7, 10293 (2016).

[31] R Tomasello, V. Puliafito, E. Martinez, A. Manchon, M. Ricci, M. Carpentieri and G. Finocchio, "Performance of synthetic antiferromagnetic racetrack memory: domain wall versus skyrmion" Journal of Physics D: Applied Physics. Vol 50. N. 32 (2017).

[32] X. Zhang M. Ezawa and Y. Zhou, "Thermally stable magnetic skyrmions in multilayer synthetic antiferromagnetic racetracks" Phys. Rev. B 94, 064406 (2016).

[33] J. Barker and O. A. Tretiakov, "Static and Dynamical Properties of Antiferromagnetic Skyrmions in the Presence of Applied Current and Temperature" Phys. Rev. Lett. 116, 147203 (2016).

[34] X. Zhang, Yan Zhou and Motohiko Ezawa, "Antiferromagnetic Skyrmion: Stability, Creation and Manipulation" Sci. Rep. 6, 24795 (2016).

[35] C. Jin, C. Song, J. Wang, and Q. Liu, "Dynamics of antiferromagnetic skyrmion driven by the spin Hall effect" Appl. Phys. Lett. 109, 182404 (2016).

[36] E. Martinez, S. Emori, N. Perez, L. Torres, and G.S.D. Beach, "Currentdriven dynamics of Dzyaloshinskii domain walls in the presence of inplane fields: Full micromagnetic and one-dimensional analysis" J. Appl. Phys. 115, (2014).

[37] J. Torrejon, E. Martinez, and M. Hayashi, "Tunable inertia of chiral magnetic domain walls" Nat. Commun. 7, 13533 (2016).

[38] S.S.P. Parkin and D. Mauri, "Spin engineering: Direct determination of the Ruderman-Kittel-Kasuya-Yosida far-field range function in ruthenium" Phys. Rev. B 44, 7131 (1991).

[39] E. Martinez, L. Lopez-Diaz, L. Torres, C. Tristan, and O. Alejos, "Oscillator based on pinned domain walls driven by direct current" Phys. Rev. B - Condens. Matter Mater. Phys. 75, 1 (2007).

[40] E. Martinez, "The stochastic nature of the domain wall motion along high perpendicular anisotropy strips with surface roughness" J. Phys. Condens. Matter 24, 24206 (2011).

[41] COMSOL Multiphysics. www.comsol.com.

[42] A. Vansteenkiste, J. Leliaert, M. Dvornik, M. Helsen, F. Garcia-Sanchez, and B. Van Waeyenberge, "The design and verification of MuMax3" AIP Advances 4, 107133 (2014). 